\title{
Towards 6G wireless networks-challenges and potential technologies
}

\author{
Rezwana Ahmed ${ }^{1}$, Mohammad Abdul Matin $^{1}$
}

\begin{abstract}
As 5G technology is advancing towards its final phase of development and the deployment of $5 \mathrm{G}$ networks is underway, academic, development and industrial communities are already moving towards the research and development of $6 \mathrm{G}$ wireless networks. While 5G technologies had been hauled as an enabler for Internet of Everything, many limitations of such cellular systems are coming to light as they are being deployed. These drawbacks of 5G networks have motivated worldwide interest on developing the next generation wireless system, $6 \mathrm{G}$, with the capability to fully incorporate wide-ranging applications from virtual reality to autonomous systems. In this paper, an overview of the first five generations of wireless systems has been shown, followed by a survey on $6 \mathrm{G}$ wireless network along with a discussion on the possible requirements and challenges of $6 \mathrm{G}$.
\end{abstract}

Keywords: 6G networks, artificial intelligence (AI), machine learning (ML), THz Communications, visible light communications (VLC), Quantum communications

\section{Introduction}

The fifth generation of mobile communication networks promises radical improvement in data transmission rates, reliability, latency and energy efficiency. This has given rise to new services classes such as enhanced mobile broadband (eMBB), massive machine-type communications (mMTC), and ultra-reliable low-latency communications (URLLC). Despite such advances, there still remain limitations that require further evolution of wireless technologies in order to meet rising need for lower latencies, higher data transmission rates and better relia- bility as the world moves towards intelligent information driven society.

As 5G enters its final phase and deployment has been initiated, academic bodies, regulatory and industrial communities have already started to research and develop future $6 \mathrm{G}$ wireless networks. It is predicted that the next ten years, 2020-2030, will be dedicated to the development of such networks. The four common underpinning themes of $6 \mathrm{G}$ are [1]-[3]:

- Transition from radio to sub-Terahertz and optical spectra

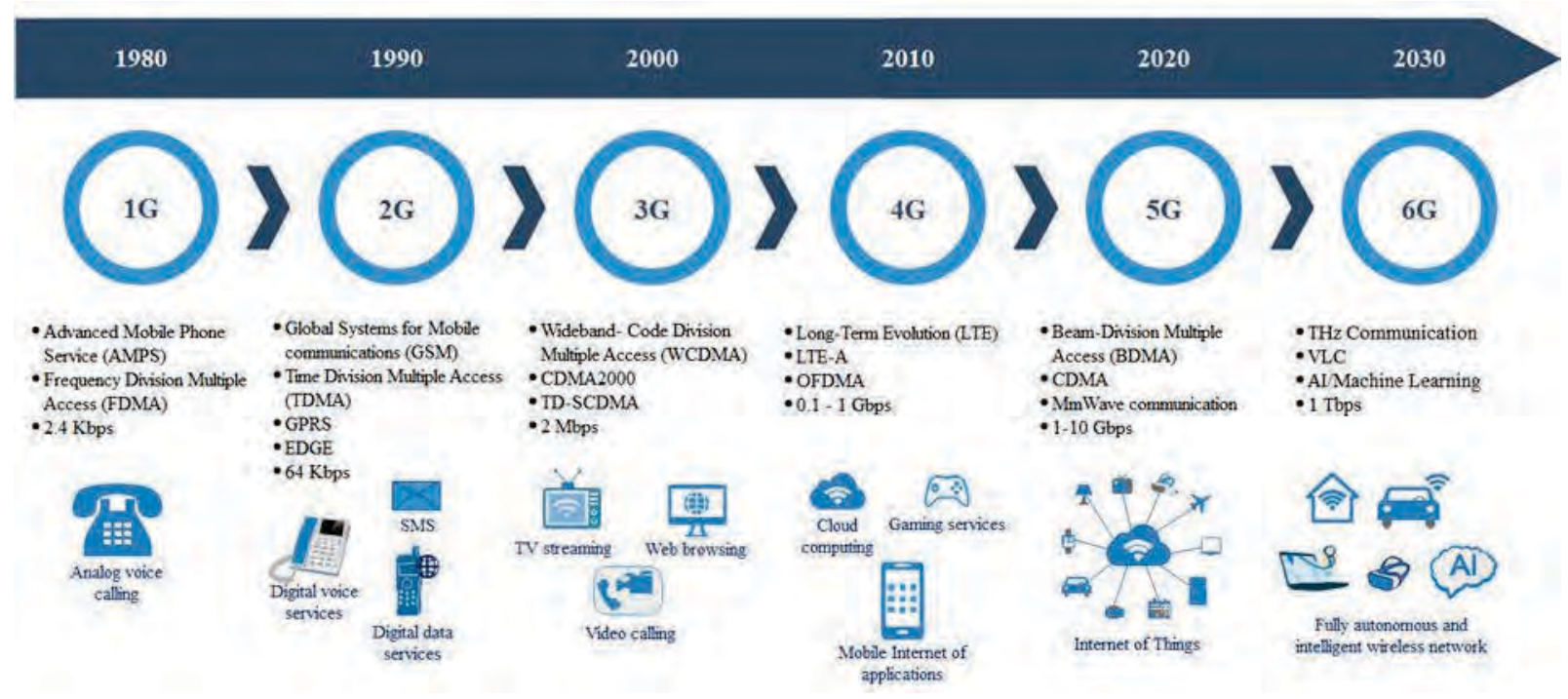

Fig. 1. Wireless evaluation $\operatorname{roadmap}(1 \mathrm{G}-6 \mathrm{G})$

\footnotetext{
${ }^{1}$ Department of Electrical and Computer Engineering, North South University, Dhaka Corresponding author: matin.mnt@gmail.com
} 
- Use of artificial intelligence (AI) and machine learning (ML) to develop autonomous networks

- integration of networks

- new and innovative applications.

In this paper, a roadmap starting from the very first communication system, the analog $1 \mathrm{G}$ system, to the latest mobile communication system extended to $5 \mathrm{G}$ network systems has been shown. The limitation of the latest communication system is highlighted to justify the necessities of $6 \mathrm{G}$ wireless networks. This paper further discusses the potential requirements of such a network, the possible techniques and what challenges researchers might face during the development of $6 \mathrm{G}$. We give an overview of the first generation of mobile networks to the fifth generation, present the limitations of $5 \mathrm{G}$ and puts forth the idea of $6 \mathrm{G}$ as a solution to the limitations of 5G. After elaborating the requirements, usage scenarios and key challenges of $6 \mathrm{G}$ that might be faced during its development, we illustrate potential technologies for $6 \mathrm{G}$ with some concluding remarks.

\section{Evolution from $1 \mathrm{G}$ to $5 \mathrm{G}$}

Wireless communication has seen unprecedented success and, in such a small period, has managed to capture the interest of billions of users. The first analog communication system was deployed in the 1980s, and from then a new generation of mobile communication system has been developed and introduced within the next ten years approximately [4]. The advancement of each generation is a complex process and often adapting different standards, technologies, and varying capacities [5].

\subsection{First generation $(1 G)$}

During the first generation period, monopoly operators owned by the state offered telecommunication. These operators usually did not have to buy spectrum and received it free of charge. Compared to today, phone calls, mobile equipment, and subscriptions were much more expensive. As a result, only around $10 \%$ of the worldwide population used these services. Voice calling was provided as the basic service and the quality of the voice calls and network coverage were excellent. Some data transmission was possible with the help of acoustic routers and fax service was also provided.

\subsection{Second generation (2G)}

The $2 \mathrm{G}$ era saw more competing systems than $1 \mathrm{G}$, for example, the IS95 in the United States and the Personal Digital Cellular (PDC) in Japan. GSM, with higher than $90 \%$ of the market share, was the dominating system standard. This huge success of GSM can be contributed to the process of competition and regulations that was introduced during the $2 \mathrm{G}$ era. The marketplace was later authorized for additional operators so that there were three or four operators in each country. Starting with only voice calls, SMS and MMS services were also added after some time. Unlike $1 \mathrm{G}, 2 \mathrm{G}$ operated using digital modulation schemes such as CDMA and TDMA. Digital data services of around $9.6 \mathrm{~kb} / \mathrm{s}$ were also provided which increased to around $35 \mathrm{~kb} / \mathrm{s}$ with the introduction of GPRS (General Packet Radio System). Ultimately, a speed of $200 \mathrm{~kb} / \mathrm{s}$ could be reached once EDGE (Enhanced Data Rates for GSM Evolution) had been introduced.

\subsection{Third generation $(3 G)$}

In 2000, a third generation of mobile communication system was proposed, the goal of which was to provide high speed transmission of data. A data transmission rate of $2 \mathrm{Mb} / \mathrm{s}$ can be reached in $3 \mathrm{G}$ networks along with fast access to the Internet. As a result, advanced services such as TV streaming, web browsing, navigational maps etc. are supported by $3 \mathrm{G}$ which were not possible in case of $1 \mathrm{G}$ and 2G. The organization 3GPP (Third Generation Partnership Project) was formed in order to establish technical specifications and define mobile systems and standards so that global roaming was possible. In Europe, this was known as UMTS (Universal Mobile Telecommunication System) a TSI driven 3G telecommunication system that used WCDMA as its air-interface technology. The American 3G variant was called CDMA2000, while ITUT named it IMT2000, which has acknowledged a novel 3G standard from China, the TD-SCDMA.

\subsection{Fourth generation $(4 G)$}

The fourth generation communication system is an IP based network system and was introduced at the end of the previous decade, during the late 2000s. The main objective of the $4 \mathrm{G}$ technology was to provide the users with high speed, high capacity and quality, security, while also making sure to provide voice and data services, internet, and multimedia services over IP at a low cost. An uplink speed of $500 \mathrm{Mb} / \mathrm{s}$ and a downlink speed of $1 \mathrm{~Gb} / \mathrm{s}$ is possible in $4 \mathrm{G}$ networks. $4 \mathrm{G}$ has increased spectral efficiency while also decreasing latency. One of the key factors of $4 \mathrm{G}$ is terminal mobility, which indicates automatic roaming across various wireless networks. This is necessary for $4 \mathrm{G}$ to provide its wireless services anywhere and at any time. Through the integration of various future and existing wireless technologies, such as OFDM, LAS-CDMA etc. $4 \mathrm{G}$ technologies ensure that uninterrupted roaming between different technologies is possible while also delivering freedom of movement. WiMAx (Wireless Interoperability for Microwave Access) and LTE (Long Term Evolution) are considered to be $4 \mathrm{G}$ technologies.

\subsection{Fifth generation $(5 G)$}

$5 \mathrm{G}$ mobile communication networks are expected to be done with standardization processes, construction of their hardware facilities and initial tests soon, after which they will be deployed for commercial use. Ensuring groundbreaking advances in data transmission rates, reliability, massive connectivity, latency and energy efficiency is one of the main goals of 5G [6]. As 
a result of using the new microwave band of the spectrum (3.3-4.2 GHz) along with the first-time innovative utilization of the millimeter-wave band, data rates can be increased greatly with speeds reaching up to $10 \mathrm{~Gb} / \mathrm{s}$. Advanced access technologies such as BDMA (Beam Division Multiple Access) and FBMC (Filter Bank Multicarrier) are employed in 5G . Usage scenarios of $5 \mathrm{G}$ can be categorized into three groups, which are eMBB (enhanced mobile broadband), mMTC (massive machine-type communications), and URLLC (ultrareliable low-latency communications). Some technologies of $5 \mathrm{G}$ are the millimeter-wave (mmWave) communications, NOMA (non-orthogonal multiple access), massive MIMO (multiple-input multiple output), scalable OFDM (orthogonal frequency division multiplexing), ultra-dense heterogeneous networks etc. 3GPP (third generation partnership project) Release 15 is the first among $5 \mathrm{G}$ standards and will be employed in 2020. The eMBB scenario of $5 \mathrm{G}$ networks is highlighted by this standard where the key measure is the data rate. For mMTC, the standard simply reuses LTE based NB-IoT and eMTC in 3GPP Release 14. As a result, for URLLC, the latency and reliability can only be as good as that ensured in case of eMBB [7].

\section{Driving force to move towards $6 \mathrm{G}$}

\subsection{Limitations of $5 G$}

Even though 5G communication systems have the capability to support URLLC, the short-packet, sensingbased URLLC functions of $5 \mathrm{G}$ means that there are limitations to how well $5 \mathrm{G}$ can provide services that require low-latency and high-reliability while ensuring high data rates as well. Thus applications such as the multisensory XR applications (AR/MR/VR) that necessitate such high requirements are unable to reach their full potential in $5 \mathrm{G}$ cellular systems. Moreover, the convergence of functions such as communication, control, computing, sensing is a requirement when it comes to upcoming applications of Internet of Everything (IoE). This aspect has been mostly neglected in $5 \mathrm{G}$. In order for the successful operation of IoE services such as autonomous systems, the network needs to provide heterogeneous devices with high data rates and reliability, low latency for both uplink and downlink communications [8]. To get past these drawbacks, a disruptive $6 \mathrm{G}$ wireless network is needed that has been designed while keeping in mind the requirements of such applications and all associated trends in technology that might arise from these emerging IoE services.

\subsection{Potential Applications}

\subsubsection{Extended Reality (X R)}

$6 \mathrm{G}$ will have the capacity to enable many XR applications that are not possible in $5 \mathrm{G}$ networks due to the inability of $5 \mathrm{G}$ systems to provide low enough latencies that will support these data rate intensive applications.
These XR applications require URLLC and eMBB while also incorporating perceptual factorsoriginating from the users cognition, senses, and physiology [8] for a truly immersive experience which $6 \mathrm{G}$ will need to be able to support. Only then can all sensory inputs be captured to provide a fully immersive XR application.

\subsubsection{Connected robotics and autonomous systems (CRAS)}

The upcoming deployment of CRAS, eg autonomous cars, drone-delivery systems, autonomous robotics etc is one of the main drivers for $6 \mathrm{G}$ wireless networks. Such systems require very low latency, for example, for public and vehicle safety in case of autonomous vehicles [9], and even eMBB transmissions for obtaining high definition maps. These strict requirements of data rate, latency and reliability is not yet provided by $5 \mathrm{G}$, and thus might influence the development of $6 \mathrm{G}$ networks. Moreover, applying machine learning techniques can assist in vehicular networks to undertake more informed and data-driven decisions in order to acquire autonomy [10]. Since AI, and especially $\mathrm{ML}$, is proposed to be a key technology of $6 \mathrm{G}$, such autonomous vehicular networks will have a significant influence on the design and development of $6 \mathrm{G}$.

\subsubsection{Haptic communications}

Haptic communication implements the sense of touch and offers an extra dimension to traditional audio visual communications in order to provide complete control and immersive steering in remote environments [11], and is an important factor in the actualization of the vast potential of VAR [12]. The features of $6 \mathrm{G}$ wireless networks will ensure that the requirements of haptic applications and systems are met, and networks by 2030 are expected to carry out haptic communications [13].

3.2.4 Wireless brain-computer interactions (B C I)

While BCI applications have been restricted to use in healthcare scenarios for the operation of prosthetic limbs or control of computing devices through the application of brain implants, the advances in implants and wireless brain-computer interfaces will mean that new applications of such technologies will arise which will require $6 \mathrm{G}$ in order to operate. These services require performance metrics fundamentally different to those provided by $5 \mathrm{G}$, and thus $6 \mathrm{G}$ wireless networks with their high rates and reliability, extremely low latency will be needed to fulfill these requirements [6]. 


\subsubsection{Automation and manufacturing}

Industry 4.0 aims to make industrial processes require as little human intervention as possible through the employment of automatic control systems and available communication technologies. Such industrial control networks need very high data rates for real-time data transfer and delay jitter as low as in the order of $1 \mathrm{~s}$ [14]. $6 \mathrm{G}$ networks will be able to provide the high reliability and data rates, and low latency these complex processes require.

\subsubsection{Blockchain and distributed ledger technologies (D L T)}

Blockchain and DLT will be transformative for IoE technologies [15]. These blockchain and DLT applications are seen as distributed sensing services as well as security measures to a certain degree [16] for the next generation. These services will require low latency, scalability and a reliable connection to carry out and thus facilitate the establishment of $6 \mathrm{G}$ wireless networks.

\subsection{7 $\mathrm{Sm}$ art $\mathrm{c}$ ity}

The concept of 'smart city' represents a future intelligent city which works towards increasing the quality of life of its inhabitants by providing them with new and diverse services with the help of the latest technologies. A key objective of $5 \mathrm{G}$ is IoT through which this concept can become reality [17]. However, such cities will only be smart in fragments, that is, different components of the city, for example, healthcare, infrastructures, vehicular networks etc. while smart will be regarded separately. On the other hand, in 6G, IoT and AI (especially ML) are said to be key enablers to realize a green smart city that is affordable and truly smart [18], [19].

\subsubsection{Holographic communication}

Present techniques of remote human interactions are expected to become outdated within the next 10 years with the rise of new innovative methods of interaction with remote environments that are truly immersive. Communications and services are predicted to integrate information from all human senses and used alongside holographic communication to support a fully immersive experience. Such holographic communications require multiple-view cameras and will need exceptionally high data rates to function properly which can be provided by $6 \mathrm{G}$ [20].

\section{Sixth generation (6G) wireless networks}

\section{$4.16 G$ vision}

As the commercial deployment of $5 \mathrm{G}$ is imminent, researchers have already begun working on developing $6 \mathrm{G}$, which can be expected to be deployed around 2030. In 2030, the intelligent information society is expected to be exceedingly digitized, and highly intelligent. An unlimited and near-instant full wireless connectivity is predicted to be an enabler of such a society. $6 \mathrm{G}$ will play a transformative role and reshape the wireless evolution towards connected intelligence from connected things [18]. 6G is envisioned to connect everything and offer full wireless connectivity which will give rise to a wide range of possible applications such as communication, control, sensing, imaging, positioning etc. [21]. Moreover, with the introduction of $6 \mathrm{G}$, data transmission rates are predicted to be increase up to $1 \mathrm{~Tb} / \mathrm{s}$, and latency to fall in the range of microseconds. Use of energy harvesting technologies and implementation of new materials will result in a system that is also energy efficient and help towards the actualization of sustainable green network [5].

\section{$4.26 \mathrm{G}$ requirements}

To address the possible future applications and challenges, 6G wireless networks will need to fulfill some of the requirements which are listed below [1]-[3], [18]:

- Extremely high data rates with a peak data rate of around $1 \mathrm{~Tb} / \mathrm{s}$ at the very least.

- $6 \mathrm{G}$ is predicted to offer user experienced data rate of 1 $\mathrm{Gb} / \mathrm{s}$ which is much larger than that afforded by $5 \mathrm{G}$. In cases like indoor hotspots, the data rate is expected to be as high as $10 \mathrm{~Gb} / \mathrm{s}$.

- High mobility of more than $1,000 \mathrm{~km} / \mathrm{h}$ and very lowlatency over the air (around 10 to $100 \mu \mathrm{s}$ ). These properties will ensure QoE for airline systems etc.

- Connectivity density is expected to be much higher compared to $5 \mathrm{G}$ with predicted connectivity density in case of hotspots to rise up to 107 devices per square kilometer and $1 \mathrm{~Gb} / \mathrm{s}$ per square meter space traffic capacity.

- Energy efficiency will be very high and the network will have to have the capability to maintain batteryfree IoT devices.

- The network is expected to work with much broader frequency bands ( $e g$, in the range $73 \mathrm{GHz}-140 \mathrm{Ghz}$, also $1 \mathrm{THz}-3 \mathrm{THz})$.

- A full coverage will be required through the implementation of ubiquitous always-on ultrabroadband network available globally using terrestrial, satellite, as well as sea domains.

- Convergence of functionalities like control, computing, sensing and communication will be necessary for the realization of a multipurpose system that will support more wide-ranging applications and services.

- Distributed and ubiquitous intelligence and computing starting from the application layer all the way to the physical layer will also be a requirement. A potential 6G architecture has been depicted in Fig. 2. 


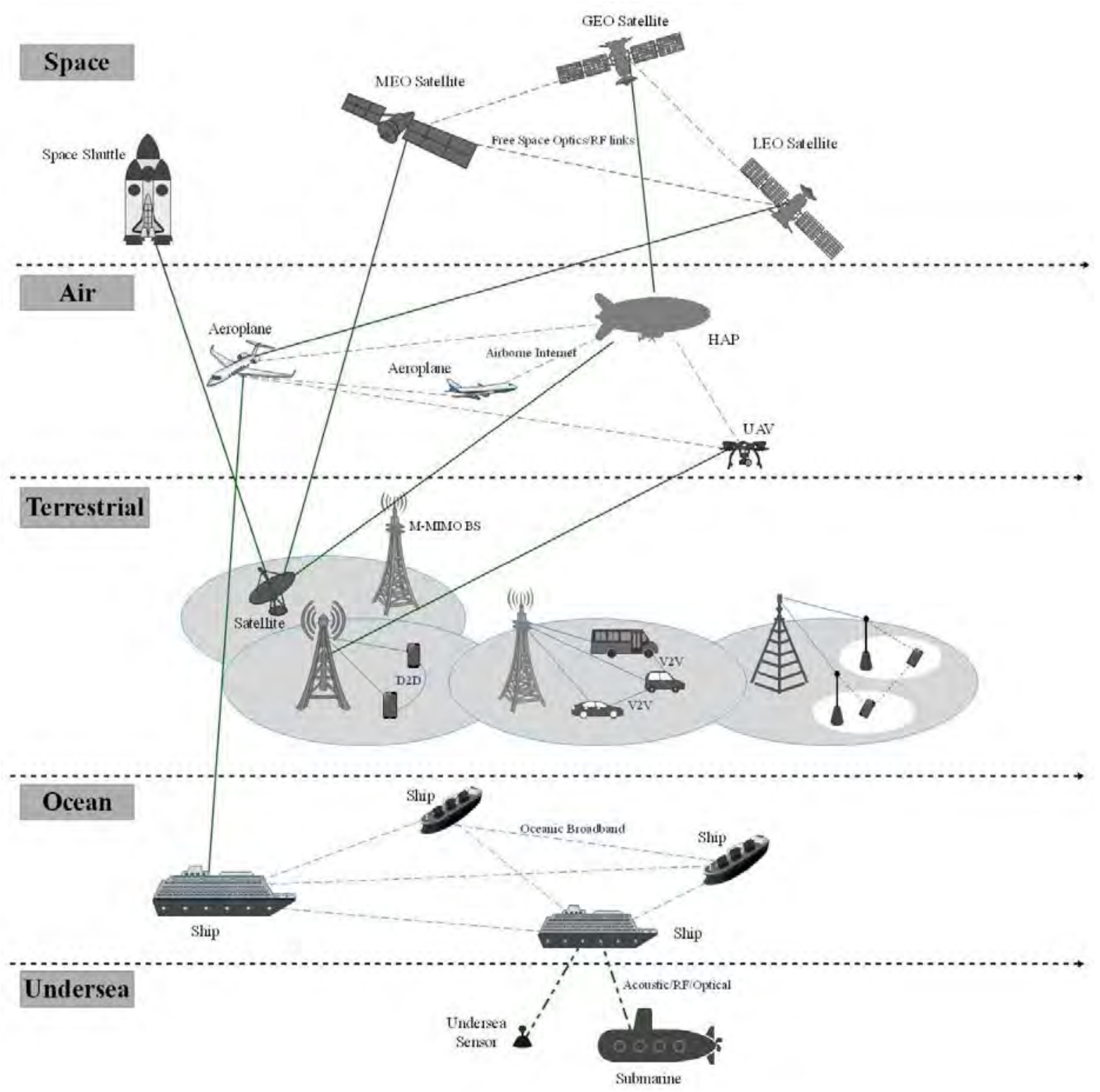

Fig. 2. Potential architecture of $6 \mathrm{~g}$

\subsection{Challenges for $6 G$}

While 6G brings with it numerous exciting applications and services, designing a network at such high frequencies has multitudes of roadblocks and challenges that researchers have to tackle in order to develop a wireless network that can deliver on its promises and meet the expectations. Some of these challenges are discussed below.

\subsubsection{Design of transceiver front-end}

At $\mathrm{THz}$ frequencies, RF amplifiers might not be physically small enough for them to be located behind antenna elements. As a result, hybrid beamforming approaches could be needed in case of practical implementations. Performance metrics of transceivers like noise figure, peak out power and linearity of LNAs continue to deteriorate with the increase of operating frequency. Moreover, phase noise increases with increase in frequency. This makes providing good performance at these high frequencies challenging, which also makes carrying out higher-order modulations difficult. In [21], the authors suggest the use of a new technique of using spatially-oversampled antenna while also presenting novel architectures of phased array devices and approaches to compact computation, all of which enable the steering of the beam using much lower chip real estate and power.

\subsubsection{Material and components}

The type of materials and components being used determine how well the multi-antenna arrays used for THZ communication will work. Materials properties and the process of fabrication used for them for frequencies above 
$50 \mathrm{GHz}$ might pose challenges [22]. This is because of the extrinsic and intrinsic composition-structure-property relationships as at such high frequencies where the wavelength is roughly the same size of the materials constituents; the materials are affected more by these frequencies. Although new research has shown that new nanomaterials such as graphene can be used to design components suitable for terahertz communication [23] such as graphene-based antennas [24] that support large bandwidth while also ensuring that the antenna efficiency is not significantly compromised.

\subsubsection{Embedded systems and software}

The embedded systems and software of the transceivers and other devices in the $6 \mathrm{G}$ wireless network will need radical changes in order to fulfill the URLLC requirements [22]. Applications of $6 \mathrm{G}$ require high throughput distributed local computing networks as well as transport lasting less than a millisecond so that there is no reactivity trade-offs resulting from multiple hops. Interplay between bandwidth, protocol, transceiver architectures and latency necessitates the use approaches that utilize highly parallel designs from wireless path to the transceiver or devices hardware and software in order to ensure reliable communications.

\subsubsection{Protocol design}

Unlike $5 \mathrm{G}$, development of $6 \mathrm{G}$ will need drastic new protocol designs. While $5 \mathrm{G}$ uses rigid frame structures and network parameters that have been pre-determined, $6 \mathrm{G}$ requires the introduction of new protocols that are AI-driven for co-ordination, scheduling and signaling purposes [8]. They need to have the ability to adapt to current as well as projected conditions of the wireless environment. Designing novel multiple access protocols are capable of choosing what multiple access technique is to be used according to network conditions and application requirements [25]. As $6 \mathrm{G}$ system will be a three dimensional network with variety of mobile devices, the need for new handover protocols accounting these characteristics might also arise. With the increase of the different kinds of wireless devices expected to be a part of the $6 \mathrm{G}$ system, identification and authentication protocols that address the issue will have to be designed as well. Moreover, all these protocols must be distributed and possess the ability to control the distributed datasets across the $6 \mathrm{G}$ network edge [8].

\section{Potential technologies for $6 \mathrm{G}$}

\subsection{THz Communications}

In order to increase bandwidth and data rates, $6 \mathrm{G}$ is predicted to use the $\mathrm{THz}$ band, available bandwidth which is of thousand times higher that of mmWave band. $\mathrm{THz}$ communication systems are expected to provide in the range of $100 \mathrm{~Gb} / \mathrm{s}$ or even higher. Since $\mathrm{THz}$ communication having available bands of more than tens of
$\mathrm{GHz}$, employing short pulse duration and narrow beam ensures that the possibility of eavesdropping is greatly reduced. The fact that $\mathrm{THz}$ waves can propagate certain materials without suffering from much attenuation can be used in special cases to benefit users. Moreover, since THz communications employ highly directional transmission, inter-cell interference can be decreased while decreasing the chances of eavesdropping as well and thus providing security.

\subsection{Visible light communications}

One of the complementary technologies of RF-based mobile communications is the OWC (Optical Wireless Communications). VLC (visible light communications) uses LED in order to serve the dual purpose of high-speed data transmission as well as lightning. Employing VLC in short-range links gives many advantages over traditional radio communications. Some of these are [5]

- Spectrum of visible light provides extremely high bandwidth (in the range of $\mathrm{THz}$ ). As a result, the spectrum is unlicensed and free.

- Visible light, the transmission medium used by VLC for sending and receiving of information, is unable to travel through opaque surfaces. Thus, information transmission networks are bounded to individual buildings and the signals cannot be picked up by receivers outside the buildings. This ensures security during data transmission, while avoiding inter-cell interference.

- As VLC uses sources of illumination as its base stations, no costly constructions of base stations or expensive maintenance is needed.

- As VLC does not employ electromagnetic waves, it is immune to outside electromagnetic interferences.

As LED lamps become more and more efficient with increasing life span, and other related technologies advances, VLC is envisioned to provide data rates in the range of hundreds of gigabits per second or even reach terabits per second speed when $6 \mathrm{G}$ gets deployed.

\subsection{Molecular communications}

Many scenarios make common wireless communications using EM waves to be inefficient or not feasible, for example the nanonetwork inside a persons body. For such cases molecular communication can be employed. MC employs molecules and biochemical signals for transmission of information in a similar manner to how living cells communicate [26]. Using advanced nanotechnology, nanodevices can be manufactured for use in biomedicine and nanoscale [27], [28]. The nanodevices can also be connected to the internet and formed into networks to enable effective transmission of information and communications. These nanodevices can then be used in the delivery of medicine in the blood vessels and monitoring body organ vitals. 


\subsection{Artificial intelligence}

Artificial Intelligence (AI), mainly machine learning $(\mathrm{ML})$, are already being used in $5 \mathrm{G}$ networks as well as in many of the applications we use on our mobile devices today. AI has the ability to learn, make predictions and take decisions through the utilization of big data training which allows AI the prospect of transforming and replacing fields that demand models and expert knowledge in wireless networks. As $6 \mathrm{G}$ wireless networks are envisioned to be intelligent and data-driven, AI is considered a key enabling technology for $6 \mathrm{G}$ autonomous networks and big data techniques are expected to be employed in $6 \mathrm{G}$ as well. An AI-driven approach and a thorough consideration of all prospective uses of AI must be taken during the design of $6 \mathrm{G}$ networks in order to realize the vision of an intelligent network.

A complex and heterogeneous network such as 6G will require an AI paradigm which is self-aware, self-adaptive, proactive, and prescriptive in order to make the network intelligent [29]. Machine learning will help to facilitate these self-organization strategies. Moreover, deep neural networks or DNNs will enable parameterization of numerous network functions with only a small set of parameters after the necessary training and can thus act as universal function approximators. As a result, a generalpurpose architecture can be used to implement functionalities throughout a network through the help of offline training algorithms to update the parameters. Such a network will have the capability to dynamically orchestrate network features in accordance to the ever changing demands of various applications and services, monitor the network status and the key performance indicators in real time to make sure the network health is maintained and the expected QoE is being provided.

\subsection{Quantum communication}

Quantum communication is expected to be another promising technique of $6 \mathrm{G}$. This communication paradigm promises very high security as information in quantum communication is encoded in quantum state through quantum particles that make it impossible for eavesdropping on the information being transferred without detection [30], [31]. Quantum communication also enables global scale long-distance communications using quantum repeaters since quantum repeaters have the ability to correct operation errors and photon loss while also segmenting the distance into intermediate segments.

\section{Conclusion}

In this paper, an overview of $6 \mathrm{G}$ was provided, along with its potential use cases and what requirements this next generation of wireless system might need in order to fulfill the future expectations. Furthermore, several promising techniques of $6 \mathrm{G}$ wireless system were elaborated upon. $6 \mathrm{G}$ communication is envisioned to be transformative and take radical steps forward technically acting as a key enabler to the advancement of society towards a truly digitized future. While possible applications of $6 \mathrm{G}$ are numerous challenges regarding electrical components, materials used, designing new architecture and protocols cannot be discarded. Worldwide cooperation amongst both academic researchers and industrial communities are necessary to tackle these challenges.

\section{REFERENCES}

[1] B. Zong, C. Fan, X. Wang, X. Duan, B. Wang, and J. Wang, "6G Technologies: Key Drivers, Core Requirements, System Architectures, and Enabling Technologies", IEEE Vehicular Technology Magazine, vol. 14, no. 3, pp. 18-27, Sept. 2019.

[2] Z. Zhang et al "6G Wireless Networks: Vision, Requirements, Architecture, and Key Technologies", IEEE Vehicular Technology Magazine, vol. 14, no. 3, pp. 28-41, Sept. 2019.

[3] E. Calvanese-Strinati et al "6G: The Next Frontier: From Holographic Messaging to Artificial Intelligence Using Subterahertz and Visible Light Communication", IEEE Vehicular Technology Magazine, vol. 14, no. 3, pp. 42-50, Sept. 2019.

[4] M. A. Matin, ed. Handbook of Research on Progressive Trends Wireless Communications and Networking, IGI Global, 2014.

[5] T. Huang, W. Yang, J. Wu, J. Ma, X. Zhang, and D. Zhang, "A Survey on Green 6G Network: Architecture and Technologies", IEEE Access, vol. 7, pp. 175758-175768, 2019.

[6] M. Sha, A. F. Molisch, P. J. Smith, T. Haustein, P. Zhu, P. De Silva, F. Tufvesson, A. Benjebbour, and G. Wunder, "5G: A tutorial overview of standards, trials, challenges, deployment, and practice", IEEE J. Sel. Areas Commun., vol. 35, no. 6, pp. 1201-1221, June 2017.

[7] L. Zhang, Y. Liang, and D. Niyato, "6G Visions: Mobile ultra-broadband, super internet-of-things, and artificial intelligence", China Communications, vol.16, no. 8, pp.1-14, August 2019.

[8] W. Saad, M. Bennis, and M. Chen, "A Vision of 6G Wireless Systems: Applications, Trends, Technologies, and Open Research Problems", IEEE Network, vol.34, no. 3, pp. 134-142, May/June 2020.

[9] H. Ullah, N. Gopalakrishnan Nair, A. Moore, C. Nugent, P. Muschamp, and M. Cuevas, "5G Communication: An Overview of Vehicle-to-Everything, Drones, and Healthcare Use-Cases", IEEE Access, vol. 7, pp. 37251-37268, 2019.

10] H. Ye, L. Liang, G. Ye Li, J. Kim, L. Lu, and M. Wu, "Machine Learning for Vehicular Networks: Recent Advances and Application Examples", IEEE Vehicular Technology Magazine, vol. 13, no. 2, pp. 94-101, June 2018.

[11] E. Steinbach et al "Haptic Communications", Proceedings of the IEEE, vol. 100, no. 4, pp. 937-956, April 2012.

[12] E. Bastug, M. Bennis, M. M edard, and M. Debbah, "Toward interconnected virtual reality: Opportunities, challenges, and enablers, ", IEEE Commun. Mag., vol. 55, pp. 110-117, June 2017.

[13] R. Li, "Network 2030: Market drivers and prospects, ", Proc. 1st International Telecommunication Union Workshop on Network 2030, New York, Oct. 2018. [Online].

[14] G. Berardinelli, N. H. Mahmood, I. Rodriguez Larrad, and P. E. Mogensen, "Beyond 5G wireless IRT for Industry 4. 0: Design principles and spectrum aspects, ", Proc. IEEE Global Communications Conf. Workshops, 2018.

[15] Y. Dai, D. Xu, S. Maharjan, Z. Chen, Q. He, and Y. Zhang, "Blockchain and Deep Reinforcement Learning Empowered Intelligent 5G Beyond", IEEE Network, vol.33, no. 3, pp.10-17, May/June 2019. 
[16] I. Ahmad, S. Shahabuddin, T. Kumar, J. Okwuibe, A. Gurtov, and M. Ylianttila, "Security for 5G and Beyond", IEEE Communications Surveys \& Tutorials, vol.21, no. 4, pp. 3682-3722, Fourthquarter 2019.

[17] S. K. Rao and R. K. Prasad, "Impact of 5G Technologies on Smart City Implementation, ", Wireless Personal Communications, vol. 100, pp. 161176, May 2018.

[18] K. B. Letaief, W. Chen, Y. Shi, J. Zhang, and Y. A. Zhang, "The Roadmap to 6G: AI Empowered Wireless Networks", IEEE Communications Magazine, vol.57, no. 8, pp. 84-90, August 2019.

[19] S. J. Nawaz, S. K. Sharma, S. Wyne, M. N. Patwary, and M. Asaduzzaman, "Quantum Machine Learning for 6G Communication Networks: State-of-the-Art and Vision for the Future", IEEE Access, vol. 7, pp. 46317-46350, 2019.

[20] A. Yastrebova, R. Kirichek, Y. Koucheryavy, A. Borodin, and A. Koucheryavy, "Future Networks 2030: Architecture \& Requirements", 2018 10th International Congress on Ultra Modern Telecommunications and Control Systems and Workshops (ICUMT), Moscow, Russia, 2018, pp. 1-8.

[21] T. S. Rappaport et al "Wireless Communications and Applications Above $100 \mathrm{GHz}$ : Opportunities and Challenges for 6G and Beyond", IEEE Access, vol. 7, pp. 78729-78757, 2019.

[22] M. Katz, M. Matinmikko-Blue, and M. Latva-Aho, "6Genesis Flagship Program: Building the Bridges Towards 6G-Enabled Wireless Smart Society and Ecosystem", 2018 IEEE 10th Latin-American Conference on Communications (LATINCOM), Guadalajara, 2018, pp. 1-9.

[23] M. Hasan, S. Arezoomandan, H. Condori, and B. Sensale-Rodriguez, "Graphene terahertz devices for communications applications", Nano Communication Networks, vol. 10, pp. 6878, December 2016

[24] M. Dashti and J. D. Carey, "GrapheneMicrostrip Patch Ultrawide Band Antennas for $\mathrm{THz}$ Communications", Advanced Functional Materials, vol. 28, no. 11, March 2018.

[25] A. S. Cacciapuoti, K. Sankhe, M. Caleffi, and K. R. Chowdhury, "Beyond 5G: THz-Based Medium Access Protocol for Mobile Heterogeneous Networks", IEEE Communications Magazine, vol. 56, no. 6, pp. 110-115, June 2018.

[26] I. F. Akyildiz, M. Pierobon, S. Balasubramaniam, and Y. Koucheryavy, "The internet of Bio-Nano things", IEEE Communications Magazine, vol. 53, no. 3, pp. 32-40, March 2015.

[27] P. Yang, Y. Xiao, M. Xiao, and S. Li, "6G Wireless Communications: Vision and Potential Techniques", IEEE Network, vol. 33, no. 4, pp. 70-75, July/August 2019.
[28] T. Nakano, Y. Okaie, S. Kobayashi, T. Hara, Y. Hiraoka, and T. Haraguchi, "Methods and Applications of Mobile Molecular Communication", Proceedings of the IEEE, vol. 107, no. 7, pp. 1442-1456, July 2019.

29] M. G. Kibria, K. Nguyen, G. P. Villardi, O. Zhao, K. Ishizu, and F. Kojima, "Big Data Analytics, Machine Learning, and Artificial Intelligence Next-Generation Wireless Networks", IEEE Access, vol. 6, pp. 32328-32338, 2018.

[30] J. Hu et al "Experimental quantum secure direct communication with single photons", Light: Science \& Applications, vol. 5, no. 9, pp. e16144-e16144, 2016.

31] N. Hosseinidehaj, Z. Babar, R. Malaney, S. X. Ng, and L. Hanzo, "Satellite-Based Continuous-Variable Quantum Communications: State-of-the-Art and a Predictive Outlook", IEEE Communications Surveys \& Tutorials, vol. 21, no. 1, pp. 881-919, Firstquarter 2019.

Received 4 August 2020

Mohammad A. Matin is a Professor of the Department of Electrical and Computer Engineering at North South University (NSU), where he has been since 2008. He has been also the coordinator of EEE program. During 2012-2017, he was an Associate Professor at UniversitiTeknologi Brunei (UTB), Brunei Darussalam. He received his BSc degree in Electrical and Electronic Engineering from BUET (Bangladesh), MSc degree in Digital Communication from Loughborough University, UK and PhD in Wireless Communication from Newcastle University, UK. He has published over 90 peer-reviewed Journal and Conference papers. He is the author/editor of 14 (fourteen) academic books such as Towards Cognitive IoT Networks (Springer, 2020), Communication Systems for Electrical Engineers (Springer, 2018), Spectrum Access and Management for Cognitive Radio Networks (Springer, 2016), Coding for MIMO-OFDM in Future Wireless Systems (Springer, 2015), Advances in Sensor Networks Research (Nova publisher, USA, 2014) and 13 (thirteen) book chapters. He serves as a referee or member of the editorial board for several international journals including IEEE Communications Magazine. He has received a number of Prizes and Scholarships including the Best Student Prize (Loughborough University), Commonwealth Scholarship, and Overseas Research Scholarship (ORS) conferred by the Committee of Vice Chancellors and Principals $(\mathrm{CVCP})$ in the UK. 